\title{
Alexithymia 3.0: reimagining alexithymia from a medical perspective
}

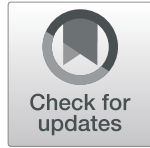

\author{
Richard D. Lane®
}

\begin{abstract}
Background: Although alexithymia research has greatly expanded in recent decades, our ability to treat it clinically remains limited. This article provides a new perspective on why that may be true and offers a fresh approach to clinical intervention.

Summary: The recent distinction between the agnosia and anomia subtypes of alexithymia, coupled with the introduction of the three-process model of emotional awareness (consisting of affective response generation, affective response representation and cognitive control), suggests that alexithymia is a phenotype that includes a spectrum of deficits that vary in their underlying neurobiology. This advance creates the opportunity to improve our ability to treat alexithymia. In the history of medicine major advances in the ability to provide effective treatments became possible once the relevant underlying morbid anatomy and physiology were discovered and the different causes of a common clinical phenotype were identified. The author suggests that we may now be entering a new era of this type in alexithymia research and clinical care. According to this perspective, Era 1.0 consisted of the pioneering clinical observations of abnormalities in emotional functioning culminating in the consensus definition of alexithymia in Heidelberg in 1976. Era 2.0 from 1976 to the present has consisted of empirical studies in which sound psychometric measures based on this clinical phenotype have been used in combination with clinical assessments and objective measures such as emotion recognition ability, peripheral physiology and neuroimaging. We may now be entering Era 3.0 in which a new model of an alexithymia spectrum grounded in brain-body interactions can transcend the constraints of a phenotype standard and provide a guide for personalized clinical care targeting the specific deficits present in a given individual. This new approach is meant to supplement rather than replace existing research and clinical practices.

Conclusion: This new era constitutes a medical perspective in three ways: 1) a focus on underlying neurobiology and associated clinical manifestations rather than an overarching phenotype; 2) a focus on the mechanisms of brain-body interactions associated with alexithymia that lead to adverse outcomes in systemic medical disorders; 3 ) clinical treatments directed at the specific deficits present in any given case.
\end{abstract}

Keywords: Alexithymia, Affective agnosia, Emotional awareness, Three-process model, Neurobiology, Psychotherapy

\section{Background}

The recent edited book on alexithymia published in 2018 [1] illustrates that alexithymia research has grown by leaps and bounds since the previous major book of its kind was published in 1997 [2]. Propelled by research

\section{Correspondence: lane@email.arizona.edu}

Department of Psychiatry, University of Arizona, 1501 N. Campbell Ave,

Tucson, AZ 85724-5002, USA using the 20-item Toronto Alexithymia Scale (TAS-20) $[3,4]$, and supplemented by a clinician-rated measure called the Toronto Structured Interview for Alexithymia (TSIA) [5], our preclinical and clinical knowledge about alexithymia has advanced considerably since a consensus definition of alexithymia was created at a conference in Heidelberg in 1976 [6] that became the theoretical foundation for these two measures. Yet, despite this progress, 
alexithymia remains a notoriously difficult phenomenon to treat clinically. Indeed, in the Forward to the 2018 book (1, pp. xii-xiii), Mark Lumley, a clinical psychologist and noted authority on alexithymia, commented that our ability to provide validated treatments for alexithymia remains seriously limited and is an important area where future advances are needed. Given the origins of the construct as a risk factor for poor physical health (and later recognized as a risk factor for poor mental health) with limited alexithymia-specific treatment options on the horizon [7], this is a serious concern and raises the issue of how significant advances can be made in clinical care.

In a recent article in this journal [8], my colleagues and I continued a line of thinking and research that predates the TAS-20 and its forerunners and potentially provides a path forward. Drawing upon clinical theories that alexithymia was a condition involving a deficit in emotional development, Gary Schwartz and I described a cognitive-developmental model of emotional awareness in 1987 that defined the developmental continuum along which an arrest in development could lead to alexithymia [9]. We created the Levels of Emotional Awareness Scale (LEAS), a performance measure of this continuum that was thought to be foundational to alexithymia but not intended to exclusively be a measure of alexithymia. The LEAS has strong psychometric properties and has been used in a series of behavioral and brain imaging studies of emotional awareness that enabled us to formulate a companion neural model $[10,11]$.

Given the importance of medical outcome prediction, and the limitations of a self-report instrument in identifying true deficits rather than beliefs about deficits [12], we were particularly interested in defining the brain basis of severe impairments in emotional awareness. After giving this considerable thought, we chose to call this condition "affective agnosia," an impairment in knowing or recognizing one's own emotions [11]. This was distinguished from an anomia, which involved an impairment in labelling or describing those emotions (an impairment that corresponds to the literal meaning of alexithymia as "difficulty expressing emotion in words"). Although problems in mental representation and description of emotion as part of alexithymia have been well-described previously [13], a key innovation here was that we proposed that different patients might have different kinds of impairments despite having the same phenotypic appearance of low emotional awareness. This was consistent with evidence from a large community sample demonstrating that two distinct clusters of individuals with high total scores on the TAS-20 could have very different characteristics [14]. Such differentiation was important because it meant that different subtypes of alexithymia could have different clinical manifestations, neural substrates, risk for clinical conditions and treatment implications. A major reason for proposing the affective agnosia and anomia subtypes was to highlight that treatment implications varied due to the underlying heterogeneity despite the phenotypic similarity. In particular, we emphasized the importance of promoting mental representation of emotion in the clinical treatment of those individuals with the most severe impairments, i.e. those with affective agnosia, and the need for different kinds of interventions in the treatment of other impairments.

To our surprise, Taylor and colleagues commented that the concept of affective agnosia was not needed and that it had already been described [13]. The details of their critique and our response can be found in a concurrent publication in this journal [8]. Their comments led us to consider how it could be that in many ways we see things so similarly and yet differ so much in our conclusions. For example, Taylor and colleagues asserted that the LEAS was not a measure of alexithymia because we did not specifically measure the classic concept of pensée operatoire by Marty and d'Muzan, which contributed to the definition of alexithymia developed in Heidelberg upon which the TAS-20 and TSIA are based and inspired the inclusion of Factor 3 "externally oriented thinking" in both. Recent work by Preece and colleagues [15], however, suggests that Factor 3 may arise from a deficit in internally-directed attention. If so, the three TAS-20 factors (in the order 3, 1, 2) correspond to difficulty in attending to, identifying and describing emotions, which would appear to map quite closely onto the processes involved in emotional awareness. A key (but not the only) difference between the measures is that the TAS-20 involves self-assessment and the LEAS is a performance measure that directly assesses the relevant ability.

In making the case that the concept of affective agnosia was needed, we pointed out that the TAS-20 has not yet been shown to be a predictor of adverse medical outcome in any large prospective epidemiological study [16]. This is a concern given the origins of the alexithymia construct in attempting to understand how psychological factors could be playing a role in medical conditions such as essential hypertension. We argued that a direct measure of a skill/ability may be better able to capture severe deficits than a self-report measure such as the TAS-20.

Since the publication of our paper on affective agnosia, we put forward the "Three-Process Model" of affective processing [17] that extends and elaborates upon the concept of affective agnosia. Specifically, we proposed that there are three inter-related (but partially dissociable) neuro-cognitive processes that can contribute to individual differences in emotional awareness - affective 
response generation, affective response representation (conceptualization), and cognitive control processes that modulate conscious accessibility - and that each can break down in multiple ways resulting in low emotional awareness. The affective agnosia model highlighted a deficit in conceptualization, the second of the three processes (resulting in a deficit in recognition, which involves linking percepts to concepts), thus putting into context how many different mechanisms could lead to a low emotional awareness phenotype. We then published a case report of a woman with severe alexithymia [18], who scored very high on the TAS-20 and very low on the LEAS (as expected given the inverse association between alexithymia and emotional awareness). In this particular case the patient showed contributions of all three elements of the three-process model to her clinical presentation. Notably the patient's ability to generate affective responses was limited, consistent with the emphasis by Bermond and Vorst [19] on the importance of assessing affective as well as cognitive components of alexithymia. The case report also illustrated how each of the three processes could be quantified in specific cases.

This line of thinking was taken a step further in a computational simulation of the three-process model, in which different components of the model can be manipulated while other elements are held constant. This simulation revealed that a low emotional awareness phenotype could be generated by seven distinct processes, each of which had specific and differentiated treatment implications [20].

Together these developments provide strong support for a view of alexithymia as a spectrum of deficits that has differential treatment implications as a function of the specific deficit in question. Thus, the concept of affective agnosia contributed to a new way of thinking about alexithymia that closely resembled the original alexithymia construct, but then introduced major new opportunities for personalized treatment of specific patients by considering the detailed attributes of each individual.

\section{Three factors or three processes?}

Bagby, Taylor and Parker drew upon well-established methods when they created a measure of alexithymia within the conceptual and measurement framework of a personality variable that varied across individuals in the population $[3,4]$. This involved assessment through selfreport, conceptualization of relevant dimensions on a continuum, relying heavily on the replicability of factor analysis as a way of validating the measurement method and being predisposed to view cutoffs on a continuum indicative of an abnormality as somewhat arbitrary and potentially ill-advised [21]. Given all of these assumptions, it follows naturally that one may study healthy individuals or patients and derive very useful information from either group as any given participant simply represents a different point on the continuum. Furthermore, personality characteristics or traits are very difficult to modify and thus, with the exception of a few disorders such as borderline personality disorder, personality is not a major focus of clinical intervention. It is not terribly surprising, then, that within this conceptual framework the treatment of alexithymia has not been a particular focus and is not particularly advanced or effective.

One may ask if alternatives are available. The approach taken by Bagby and colleagues, and by far the predominant approach in the field of personality research, is nomothetic, i.e. population-generalizable models of inter-individual variation. An alternative and valid but less commonly used approach is idiographic, i.e. person-specific models of intra-individual variation [22]. As an illustration of the difference, a study of 22 participants was conducted in which each person rated 30 personality-related adjectives 90 times on separate days using ecological momentary assessment methodology [23]. When a between-subjects (nomothetic) factor analysis was performed on the mean value of the adjectives from each subject (i.e. one value representing the 90 observations for each adjective per person), the traditional "five factor" solution was obtained corresponding to the Five Factor Model of Personality [24]. However, when the factor analyses were performed separately for each person on a within-subjects (idiographic) basis, taking all 90 observations from each person into account, none of the 22 subjects had a five-factor solution and the number of factors for any given person varied from two to four. The idiographic perspective does not rely on the concept of a continuum that compares one subject relative to another but instead examines how a combination of characteristics operate within a given person over time, helping to define the unique identity of each individual and the dynamic nature of that person's individualized treatment needs that evolve as treatment proceeds - an approach very consistent with the three-process model.

\section{The potential value and use of a medical perspective on alexithymia}

Returning to first principles, if we are interested in relating an aspect of mental/emotional functioning, in this case alexithymia, to medical outcome, we have different options in how we approach the characterization of the former variable. Instead of conceptualizing alexithymia purely from the standpoint of personality in a population-based manner, what if this predictor of medical outcome was 
considered from an idiographic perspective using the epistemological framework of a medical condition?

As an illustration of this approach, consider the example of "dropsy" or swelling of the soft tissues, a term coined in the thirteenth century (now called edema), references to which can be found in ancient Sumerian, Babylonian, Egyptian and Greek texts [25]. A true understanding of the causes of edema was not possible until William Harvey discovered the circulation of blood in the early seventeenth century [26]. Only in the late seventeenth century was the heart and impairment in its function (e.g. congestive heart failure $[\mathrm{CHF}]$ ) recognized to be an important cause of edema, but this was not widely accepted until the eighteenth century. Moreover, it would not be until the nineteenth century that cardiac and renal causes of edema were differentiated. Needless to say, our ability to treat edema in modern day medicine involves making a proper (causal) diagnosis. Surely there are treatments such as diuretics that are useful across a variety of etiologies, but more specific treatment, such as afterload reduction or cardiac resynchronization therapy for $\mathrm{CHF}$, becomes possible only when the specific cause(s) of edema can be demonstrated in a specific person. Importantly, once different etiologies are understood a more differentiated description of the pattern of edema becomes relevant, e.g. edema in the abdomen (ascites) is prominent in liver failure, edema in the lungs is prominent in CHF, but peripheral edema in the legs may be present in both. From this vantage point, it would appear to be useful to further explore the notion of different kinds of alexithymia based on our emerging understanding of the brain basis of emotional awareness and emotion processing and the different possible ways that such problems may arise. If so, it becomes possible to consider alexithymia as a spectrum of deficits arising from known anatomy and physiology, i.e. within the epistemological framework of clinical medicine. Such an approach is entirely consistent with the Research Domain Criteria project initiated by the National Institute of Mental Health in the U.S. in which underlying neurobiology contributes to the conceptualization and classification of psychopathology [27].

This line of thinking raises the question of whether a definition of alexithymia based on a clinical description of the phenomenon alone (as in the Heidelberg consensus) is still to be preferred to an updated one informed and constrained by a brain model, particularly one in which plausible autonomic and immune mechanisms that link to disease pathophysiology can be specified [11, 28]. One consequence of such a shift would be to resolve an apparent paradox that currently exists in the alexithymia literature. By basing the definition of alexithymia on the Heidelberg consensus, and using this as the foundation for the TAS-20 and TSIA, the construct of alexithymia as a multi-dimensional trait (difficulty attending to, identifying and describing one's own emotions) that varies on a continuum is derived from the original description of alexithymia as a clinical disorder. Yet, the paradox is that characterizing alexithymia as a continuum results in the frequent (but not exclusive) use of healthy subjects to study the phenomenon, and hesitation to use the continuum to define a severe subgroup with a disorder. Contrast this with the approach proposed here, which is to define the continuum (emotional awareness) based on how the system operates normatively (cf. the circulation of blood) and use this continuum to define a severe abnormality such as affective agnosia (cf. CHF). One can see how from the former perspective the notion of defining a disorder would be problematic whereas it follows naturally from the latter. Indeed, as in the case of dropsy/edema, what makes it possible to go beyond a generic clinical description and provide personalized treatment is the ability to define the underlying anatomy and physiology (cf. the cardiovascular system) and an understanding of how different underlying abnormalities can lead to the same phenotypic appearance. Indeed, a distinct benefit of this new approach is the opportunity to improve clinical care in an area where good clinical care has not yet been demonstrated (1, pp. xii-xiii). As noted by Lumley, and supported by reviews of psychotherapeutic treatment of alexithymia [29,30], alexithymia is a difficult condition to treat, there are very few studies reporting interventions that specifically target the deficits that characterize alexithymia, and none describe the kind of comprehensive and individualized treatment approach that is made possible by the 3-process model.

Importantly, different deficits in the brain-based model have different psychological meaning that can translate into different kinds of psychological interventions. Although the treatment may be psychological (e.g. improving the ability to mentalize one's emotions, or improving the ability to link bodily sensations with existing emotion concepts), the effects on underlying brain-body interactions and thus the mechanisms by which the psychological characteristic (alexithymia) confers risk for adverse medical outcomes can potentially be demonstrated and improved. This revised orientation facilitates communication with physicians who are providing the medical care for the patient in question. Consistent with a previous editorial in this journal [28], this would amount to translating the biopsychosocial model into the biomedical terms that physicians readily understand and accept.

It should be highlighted that this does not eliminate the value of self-reported assessment of deficits (TAS-20 factors 1 and 2), proclivities (TAS-20 factor 3) or beliefs but puts all such methods of assessment into the larger context of the three-process model. Surely our ability to 
define different subtypes of alexithymia in the brain is in its infancy, but perhaps this is a valuable focus for future research, as our ability to image the structure and function of the living human brain is continuously being improved as is our ability to concomitantly measure peripheral physiology in the scanning environment and thus study brain-body interactions with increasing precision.

To summarize, the key takeaway points regarding a medical perspective are that: 1) the description of clinical manifestations, including psychological characteristics, are informed and constrained by what is known about the underlying neurobiology, thus going beyond clinical description alone; this shift in focus is captured by the differences between the nomothetic three factors (of the TAS-20) and the idiographic three processes (of the brain model); 2) the underlying mechanisms in an individual case can serve as a guide for personalized treatment; 3) this in no way limits or changes the provision of psychological or behavioral treatments or the professional identity of those who provide such treatment; and 4) a definition of alexithymia based on the three-process model potentially provides a much broader (albeit more complex) approach to assessment and treatment than that based on the clinical description from Heidelberg [6] and thus also expands opportunities for research.

\section{Alexithymia $\mathbf{3 . 0}$}

We can now more formally put the current proposal into historical perspective by defining three eras of alexithymia research and clinical care. Alexithymia 1.0 would be that period in which the clinical characteristics of what came to be known as alexithymia were first observed. This would encompass the early clinical observations by Alexander, Ruesch, Marty and d'Muzan and Sifneos and Nemiah culminating in the creation of the name "alexithymia" and its definition as described by the Heidelberg consensus in 1976. The difficulty of treating the condition using traditional psychotherapeutic methods was an important reason for describing it and identifying it as a worthwhile topic of investigation. Alexithymia 2.0 would be the era between then and now in which measures based on the clinical description from Heidelberg such as the TAS-20 have been the mainstay of alexithymia research which have been used in conjunction with objective measures (e.g. emotion recognition, peripheral physiology, brain imaging) or clinical assessments of systemic medical or psychiatric disorders. As noted above, while major advances have been made, this has not included significant inroads in our ability to treat alexithymia. Alexithymia 3.0, should it take hold, and eventually become a consensus perspective in the field, would be a new era starting now in which the definition of alexithymia was based on the underlying neurobiology (the three-process model) rather than clinical description alone. With such an approach the consensus clinical description from Heidelberg would still be relevant but no longer the ultimate definition. A primary motivation for this newer approach is the new perspective it offers in treating alexithymia more definitively, consistent with the emergence of what is called "precision psychiatry" [31].

It is proposed that Era 3.0 is defined and guided by the three-process model of emotion processing consisting of affective response generation, affective response representation (conceptualization), and cognitive control processes [17]. Unlike the Heidelberg definition, the model can accommodate important subtypes of alexithymia e.g. deficits in emotion conceptualization with or without intact emotion generation. It can also distinguish between the phenotypical appearance of alexithymia (lack of apparent experience or expression of emotion) due to cognitive control mechanisms (lack of interest, lack of attention to emotion, defensive processes) and actual core deficits in the generation and/or conceptualization of emotion. Although alexithymia as a condition involving deficits is distinct from clinical conditions involving defenses, and can be conceptualized and measured as such, the clinical reality is that in real people defenses and deficits co-exist. The three-process model has the virtue of incorporating both deficits and defenses within one framework and as such opens the way to examine how defenses (and other related avoidance mechanisms) can lead to deficits and how underlying deficits can make it especially difficult to resolve conflicts that involve defenses. While it is true that separate measures of alexithymia and defenses are available to study such associations [15], the three process model has the advantage of situating such interactions within a plausible mechanistic framework. Indeed, the computational simulation of emotional awareness explored how distinct processes, including deficits and processes related to defenses (such as attention biases), can interact in a variety of ways to produce the same phenotype [20]. This innovation may also provide an opportunity for integration within the Operationalized Psychodynamic Diagnosis system [32] in which psychopathology based on conflicts and psychopathology based on deficits are two broad and non-overlapping categories.

Another advantage is that by defining the abnormality (alexithymia) in relation to a normative process (emotional awareness), it makes it possible to quantify the degree of strength on the same continuum that captures a deficit at the other extreme. Just as the absence of edema does not enable one to determine how well the heart is pumping blood (e.g. whether ejection fraction is 45 or 65), a continuum focused on difficulty can at best only 
identify the absence of difficulty. As such this approach provides a more inclusive conceptual framework that places alexithymia and aspects of emotional resilience on the same continuum.

Another important and related concept is that alexithymia has been considered a disorder of emotion regulation [2]. The fundamental idea is that if emotion is activated but the person is unaware of that, it is not possible to consciously and intentionally use emotion regulation strategies such as suppression, reappraisal or mindfulness to regulate that emotional response. The ability to be emotionally aware captures the fundamental deficit in alexithymia that interferes with conscious/ intentional emotion regulation. It is also recognized that a great deal of emotion regulation happens implicitly and without conscious awareness [33]. This is also readily addressed within a model of emotional awareness that places implicit and explicit processes on the same developmental continuum [34] and thus can explain how explicit processing modulates implicit processing. The third process, cognitive control, includes both voluntary and involuntary/automatic mechanisms. Brainbased models can explain how behaviors that serve an emotion-regulatory role can be deployed in situations without conscious awareness [35]. Thus, the abnormalities in emotion regulation that are associated with alexithymia can be addressed quite well by the three-process model.

\section{Implications}

A brain-based model offers new solutions to vexing problems that have been unresolved in alexithymia research. For example, there is a debate about how to understand alexithymia that arises developmentally (and the possible contributions of genetic factors and geneenvironment interactions), as opposed to alexithymia that has a later onset in adult life after trauma, so-called secondary alexithymia [36]. A brain-based model potentially provides a unifying framework. For example, emotional neglect in childhood is associated with a premature shift from a positive to a negative correlation in the activity of the prefrontal cortex and amygdala, which is associated with cutting short the period of emotional learning (associated with a positive prefrontalamygdala correlation) and a premature shift to independent self-regulation (associated with a negative prefrontal-amygdala correlation) [37-39]. The emotional numbing of PTSD has been shown to involve impaired activation during high arousal states of the dorsomedial prefrontal cortex [40], an area involved in mental representation of emotional states. The latter phenomenon corresponds to the intermittent loss of the capacity for mental representation of emotional states in borderline personality disorder that typically occurs in the context of hyperarousal [41]. An important area of future alexithymia research is to explore how altered prefrontalamygdala connectivity arising from early life trauma may predispose to adult onset PTSD or borderline personality disorder.

The emphasis thus far has been on how alexithymia increases risk for adverse medical outcomes, consistent with the context of the original insights by Sifneos and Nemiah [42]. An important additional thread in the alexithymia literature has been its association with various forms of psychopathology [1]. In fact, the threeprocess model provides a new way of exploring how emotional processing is altered in different forms of psychopathology. One of the advantages of this model, and the continuum from deficits to strengths, is that it provides an alternative way of characterizing limitations in emotion processing independent of defenses, which are difficult to quantify [43]. In so doing, it also provides a common framework for incorporating psychopathology into studies of the association between alexithymia and adverse medical outcomes. Recent research demonstrates that life expectancy is significantly reduced in psychiatric disorders such as major depression, bipolar disorder, schizophrenia and anxiety disorders such as PTSD independent of mortality due to suicide or accidents [44]. A likely contributing factor is the emotion dysregulation inherent in these disorders, which causes physiological wear and tear, contributes to disease pathophysiology and can be studied within the framework of the three-process model.

As stated above, the three-process model provides a mechanistic explanation for how alexithymia contributes to disease pathophysiology. This can be expanded in considerable detail using the framework of computational neuroscience as it applies to emotional awareness [45]. One of the core elements of computational neuroscience is predictive processing, which states that perceptions of the external environment are not built up from scratch but involve predictions activated by the situation that are fine-tuned by raw sensory data to correct prediction error. Just as the body like the external environment is external to the brain, perceptions of internal emotional states are influenced by predictions that are a function of the person's emotion concept repertoire. Someone with alexithymia, e.g. an under-developed repertoire of emotion concepts, will interpret bodily affective states in a coarse-grained and undifferentiated way. Strong (highly precise) priors about one's current bodily state that are not updated by prediction error due to raw interoceptive data will drive peripheral physiology in a way consistent with the metabolic demands entailed by current predictions, a phenomenon known as active inference. The failure to update predictions based on bodily feedback can lead to inaccurate or inappropriate physiological and behavioral responses. Recent theorizing 
suggests that this is a useful way to understand the contribution of impaired emotion processing to the pathophysiological basis of functional somatic syndromes and functional neurological disorders [46, 47].

Since our emphasis here is on clinical treatment of different kinds of deficits, a clinical interview using the TSIA [5] or Modified Beth Israel Hospital Questionnaire [48] should be conducted to confirm the need for treatment. Once a person is so identified, detailed assessments of each of the three processes may be completed, as noted in our companion paper [8]. This may be supplemented by structural and functional brain imaging, which lays the groundwork for eventually using imaging as a screening tool for treatment. Interventions should then be undertaken that target the specific abnormalities so identified from a three-process perspective. Follow-up studies evaluating the effect of such interventions on psychiatric and systemic medical disorder outcomes as well as brain structure and function would be needed to generate hypotheses that could be tested in subsequent larger-scale studies.

\section{Conclusion}

The purpose of this commentary has been to put the alternative perspective presented in the companion paper [8] into the broader context of the history of medicine. Clearly articulating such an alternative perspective is needed to motivate research to test its claims and implications. Without articulation of this vision in advance, such observations are not likely to be made spontaneously [49]. Era 3.0 in alexithymia research and practice is a vision that will not be firmly established until new research is conducted that better predicts outcome or serves as a better guide to clinical care than its predecessors.

This new brain-based conceptualization of alexithymia as a spectrum of deficits expands upon the pioneering clinical descriptions of an alexithymia phenotype from an era when functional and structural neuroimaging of emotional states and emotion processing had not yet become available. This conceptual transition was made possible first by recognizing that alexithymia factor 3 (externally-oriented thinking) appears to involve a deficit in internally-directed attention rather than a primary disposition for externally-oriented thinking [15], the latter thus being a result of the former. By then defining alexithymia as difficulty in attending to, identifying and describing emotion (i.e. TAS-20 factors $3,1,2$ ), it became possible to equate alexithymia with the foundational phenomenon of emotional awareness and its impairments. At that point the brain basis of emotional awareness using the "three process model" became relevant. This transition from phenotype based on clinical description to a mechanistic model of its underlying neurobiological determinants, and the associated ability to describe brain-body interactions, their relevance to systemic medical disorders and their implications for more effective treatments, was then placed in the broader historical context of the typical sequence of events in clinical medicine, while also being consistent with newer developments in the mental health field such as RDOC, in which neurobiological mechanisms are understood to constrain and influence psychological conceptualizations, and precision psychiatry, in which treatments are tailored to the specific needs of the individual. Importantly, this new perspective is meant to be broad enough to include current methods in the field, such as the TAS-20 and other self-report measures, while recognizing that in certain contexts and for certain purposes use of those alone may be incomplete.

A medical perspective advances the construct of alexithymia by moving away from exclusive reliance on clinical description to a recognition that a clinical phenotype of low emotional awareness can be due to different underlying neurobiological mechanisms, which in turn provides the basis for person-centered care that targets the specific deficits of a given individual. By advancing our understanding of the varieties of deficits that make up the alexithymia spectrum guided by the three-process model, psychological conceptualizations and interventions can follow, rather than determine, neurobiological exploration. This is especially important because we need to understand how the brain influences the etiology, onset and course of physical disease processes and we now have the opportunity to eventually link mind, brain, peripheral physiology and disease outcome in a unified model of emotion processing and its deficits.

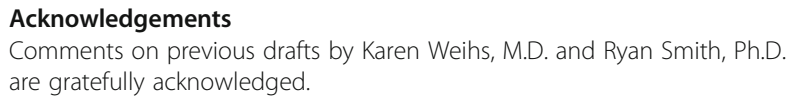

Acknowledgements

Comments on previous drafts by Karen Weihs, M.D. and Ryan Smith, Ph.D. are gratefully acknowledged.

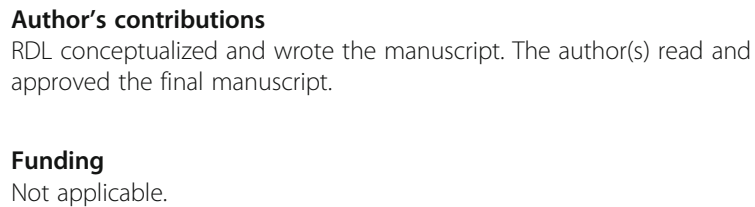

Availability of data and materials

Not applicable

Ethics approval and consent to participate

Not applicable

Consent for publication

Not applicable

Competing interests

None. 
Received: 15 May 2020 Accepted: 29 July 2020

Published online: 04 September 2020

\section{References}

1. Luminet O, Bagby RM, Taylor GJ, editors. Alexithymia: advances in research, theory, and clinical practice. Cambridge: Cambridge University Press; 2018.

2. Taylor G, Bagby R, Parker J. Disorders of affect regulation: alexithymia in medical and psychiatric illness. Cambridge: Cambridge University Press; 1997.

3. Bagby R, Parker J, Taylor G. The twenty-item Toronto alexithymia scale-l. item selection and cross-validation of the factor structure. J Psychosom Res. 1994;38:23-32

4. Bagby R, Parker J, Taylor G. The twenty-item Toronto alexithymia scale-II. Convergent, discriminant, and concurrent validity. J Psychosom Res. 1994; 38:33-40.

5. Bagby R, Taylor G, Parker J, Dickens S. The development of the Toronto structured interview for alexithymia: item selection, factor structure, reliability and concurrent validity. Psychother Psychosom. 2005;75:25-39.

6. Brautigam W, von Rad M. Editors. Toward a theory of psychosomatic disorders. Alexithymia, pensée operatoire, psychosomatisches phanomen. Proceedings of the $11^{\text {th }}$ European conference on psychosomatic research. Basel: Karger; 1977.

7. Samur D, Tops M, Schlinkert C, Quirin M, Cuijpers P, Koole SL. Four decades of research on alexithymia: moving toward clinical applications. Front Psychol. 2013;4:861.

8. Lane RD, Solms M, Weihs KL, Hishaw A, Smith R. Affective agnosia: a core affective processing deficit in the alexithymia spectrum. BioPsychoSoc Med. https://doi.org/10.1186/s13030-020-00184-w.

9. Lane R, Schwartz G. Levels of emotional awareness: a cognitivedevelopmental theory and its application to psychopathology. Am J Psychiatry. 1987;144:133-43.

10. Lane RD. Neural correlates of conscious emotional experience. In: Lane R, Nadel L, editors. Cognitive neuroscience of emotion. New York: Oxford University Press; 2000. p. 345-70.

11. Lane RD, Weihs KL, Herring A, Hishaw A, Smith R. Affective agnosia: expansion of the alexithymia construct and a new opportunity to integrate and extend Freud's legacy. Neurosci Biobehav Rev. 2015;55:594-611.

12. Nam G, Lee $H$, Lee JH, Hur JW. Disguised emotion in alexithymia: subjective difficulties in emotional processing and increased empathic distress. Front Psychiatry. 2020;11:698.

13. Taylor GJ, Bagby RM, Parker JD. What's in the name 'alexithymia'? A commentary on "Affective agnosia: Expansion of the alexithymia construct and a new opportunity to integrate and extend Freud's legacy.". Neurosci Biobehav Rev. 2016;68:1006-20.

14. Ueno M, Maeda M, Komaki G. Different subgroups of high-scorers on the TAS-20 based on the big five personality traits. Personal Individ Differ. 2014; 68:71-6.

15. Preece D, Becerra R, Allan A, Robinson K, Dandy J. Establishing the theoretical components of alexithymia via factor analysis: introduction and validation of the attention-appraisal model of alexithymia. Personal Individ Differ. 2017;119:341-52.

16. Kojima M. Alexithymia as a prognostic risk factor for health problems: a brief review of epidemiological studies. BioPsychoSoc Med. 2012;6(1):21.

17. Smith R, Killgore WD, Lane RD. The structure of emotional experience and its relation to trait emotional awareness: a theoretical review. Emotion. 2018;18(5):670.

18. Smith R, Kaszniak AW, Katsanis J, Lane RD, Nielsen L. The importance of identifying underlying process abnormalities in alexithymia: implications of the three-process model and a single case study illustration. Conscious Cogn. 2019;68:33-46.

19. Vorst HC, Bermond B. Validity and reliability of the Bermond-Vorst alexithymia questionnaire. Personal Individ Differ. 2001;30(3):413-34.

20. Smith R, Lane RD, Parr T, Friston KJ. Neurocomputational mechanisms underlying emotional awareness: insights afforded by a deep temporal active inference model and their potential clinical relevance. Neurosci Biobehav Rev. 2019;107:473-91.

21. Haslam N, Holland E, Kuppens P. Categories versus dimensions in personality and psychopathology: a quantitative review of taxometric research. Psychol Med. 2012;42(5):903-20.

22. Molenaar PC, Campbell CG. The new person-specific paradigm in psychology. Curr Dir Psychol Sci. 2009;18(2):112-7.

23. Borkenau P, Ostendorf F. The big five as states: how useful is the five-factor model to describe intraindividual variations over time? J Res Pers. 1998; 32(2):202-21.
24. McCrae RR, Costa PT. Validation of the five-factor model of personality across instruments and observers. J Pers Soc Psychol. 1987;52(1):81.

25. Eknoyan G. A history of edema and its management. Kidney Int Suppl. 1997;59:S118-26.

26. Comrie JD. Remarks on historical aspects of ideas regarding dropsy. Brit Med J. 1928;2(3527):229-32.

27. Cuthbert BN, Insel TR. Toward the future of psychiatric diagnosis: the seven pillars of RDoC. BMC Med. 2013;11:126-34.

28. Lane RD. Is it possible to bridge the biopsychosocial and biomedical models? BioPsychoSoc Med. 2014;8:3-5.

29. Ogrodniczuk JS, Kealy D, Hadjipavlou GA, Cameron K. Therapeutic issues. In: Luminet O, Bagby RM, Taylor GJ, editors. Alexithymia: advances in research, theory, and clinical practice. Cambridge: Cambridge University Press; 2018. p. 190-206.

30. Taylor G. Treatment considerations. In: Taylor G, Bagby R, Parker J, editors. Disorders of affect regulation: alexithymia in medical and psychiatric illness. Cambridge: Cambridge University Press; 1997. p. 248-66.

31. Fernandes BS, Williams LM, Steiner J, Leboyer M, Carvalho AF, Berk M. The new field of 'precision psychiatry'. BMC Med. 2017;15(1):1-7.

32. OPD Task Force, editor. Operationalized psychodynamic diagnosis OPD-2: Manual of diagnosis and treatment planning. Göttingen: Hogrefe Publishing; 2008.

33. Braunstein LM, Gross JJ, Ochsner KN. Explicit and implicit emotion regulation: a multi-level framework. Soc Cogn Affect Neurosci. 2017;12(10):1545-57.

34. Lane RD. Neural substrates of implicit and explicit emotional processes: a unifying framework for psychosomatic medicine. Psychosom Med. 2008; 70(2):214-31.

35. Smith $R$, Lane RD, Nadel L, Moutoussis M. A computational neuroscience perspective on the change process in psychotherapy. In: Lane RD, Nadel L, editors. Neuroscience of enduring change: implications for psychotherapy. New York: Oxford University Press; 2020. p. 395-432.

36. Wise TN, Mann LS, Mitchell JD, Hryvniak M, Hill B. Secondary alexithymia: an empirical validation. Compr Psychiatry. 1990;31(4):284-8.

37. Gee DG, Gabard-Durnam LJ, Flannery J, Goff B, Humphreys KL, Telzer EH, Hare TA, Bookheimer SY, Tottenham N. Early developmental emergence of human amygdala-prefrontal connectivity after maternal deprivation. P Natl Acad Sci USA. 2013;110(39):15638-43.

38. Callaghan BL, Tottenham N. The stress acceleration hypothesis: effects of early-life adversity on emotion circuits and behavior. Curr Opin Behav Sci. 2016;7:76-81.

39. Tottenham N. Neural meaning making, prediction, and mPFC-subcortical development following early adverse caregiving. Dev Psychopathol. (in press).

40. Frewen PA, Dozois DJA, Neufeld RWJ, Lane RD, Densmore M, Stevens TK, Lanius RA. Emotional numbing in PTSD: an fMRI study. J Clin Psychiat. 2012; 73(4):431-6.

41. Bateman A, Fonagy P. Mentalization-based treatment for borderline personality disorder: a practical guide. Oxford: Oxford University Press; 2007.

42. Nemiah J, Sifneos P. Affect and fantasy in patients with psychosomatic disorder. In: Hill O, editor. Modern Trends in Psychosomatic Medicine, vol. 2; 1970. p. 26-34.

43. Juni S. The defense mechanisms inventory: theoretical and psychometric implications. Curr Psychol. 1998;17(4):313-32.

44. De Hert M, Correll CU, Bobes J, Cetkovich-Bakmas MA, Cohen DA, Asai I, Detraux J, Gautam S, Möller HJ, Ndetei DM, Newcomer JW. Physical illness in patients with severe mental disorders. I. Prevalence, impact of medications and disparities in health care. World Psychiatry. 2011;10(1):52-77.

45. Smith R. The three-process model of implicit and explicit emotion. In: Lane RD, Nadel L, editors. Neuroscience of enduring change: implications for psychotherapy. New York: Oxford University Press; 2020. p. 25-55.

46. Van den Bergh O, Witthöft M, Petersen S, Brown RJ. Symptoms and the body: taking the inferential leap. Neurosci Biobehav Rev. 2017;74:185-203.

47. Edwards MJ, Fotopoulou A, Pareés I. Neurobiology of functional (psychogenic) movement disorders. Curr Opin Neurol. 2013;26(4):442-7.

48. Arimura T, Komaki G, Murakami S, Tamagawa K, Nishikata H, Kawai K, Nozaki T, Takii M, Kubo C. Development of the structured interview by the modified edition of Beth Israel hospital psychosomatic questionnaire (SIBIQ) in Japanese edition to evaluate alexithymia. Jpn J Psychosom Med. 2002;42:259-69.

49. Kuhn TS. The structure of scientific revolutions. 4th ed. Chicago: The University of Chicago Press; 2012.

\section{Publisher's Note}

Springer Nature remains neutral with regard to jurisdictional claims in published maps and institutional affiliations. 